\title{
DISCRETENESS OF SOME CONTINUOUS SPECTRUM EIGENFUNCTION EXPANSIONS
}

\author{
DON B. HINTON AND ROBERT M. KAUFFMAN
}

(Communicated by Palle E. T. Jorgensen)

\begin{abstract}
We discuss replacing the integrals in continuous spectrum expansions by finite sums, in the special case of the Dirichlet problem for secondorder ordinary differential operators on a half-line. The error is controlled in the operator norm of $B(Y, Z)$, where $Y$ and $Z$ are natural Hilbert spaces for the problem.
\end{abstract}

\section{INTRODUCTION}

Discrete spectrum and continuous spectrum eigenfunction expansions for selfadjoint operators in a Hilbert space appear to be quite different things altogether. A great deal of literature is devoted to the rate of convergence of finite sums in the discrete case, but in the continuous case the use of integral expansions is necessary; the idea of approximation by finite sums seems lost. Nonetheless, the idea of approximation by finite sums is the intuitive idea behind Fourier analysis: the approximation of arbitrary waves by standing waves. This property also seems desirable if the continuous spectrum eigenfunction expansion is to be calculated approximately on a computer. In this paper we analyze the extent to which approximation by finite sums can be rescued, in the special case of a second-order ordinary differential operator in $L_{2}[0, \infty)$.

We calculate the size of the difference between a function and its approximation by finite sums of multiples of (possibly non- $L_{2}$ ) eigenfunctions by taking the norm of this difference in a Hilbert space $Z$. To be effective in any computational scheme, the eigenfunctions used in the finite sum expansion should not depend on the function being approximated in order to get the error to within the desired accuracy. This means really that the approximation must converge in operator norm in $B(Y, Z)$, where $Y$ is another Hilbert space. We are then led to the approximation of an operator in $B(Y, Z)$ by finite sums of multiples of eigenprojections which come in some sense from the original selfadjoint operator $H$. In order for this to be possible, the operator being approximated should be some function of $H$. Also, before any analysis is possible, the term "eigenprojection", which must be an element of $B(Y, Z)$, needs to be defined rigorously.

Received by the editors February 5, 1992.

1991 Mathematics Subject Classification. Primary 47E05, 34L10; Secondary 47A70. 
In an earlier paper [7], the basic functional analytic theory of this kind of approximation was studied for a class of operators including most reasonably well-behaved selfadjoint ordinary and partial differential operators. It is not hard to see that the question for any bounded continuous function of $H$ reduces to the study of spectral projections $P(\Delta)$ for $H$, where $\Delta$ is a Borel subset of the real numbers. It was shown, under quite general hypotheses where $Y$ and $Z$ are Hilbert spaces, that the approximation property holds for $P(\Delta)$ if and only if $P(\Delta)$ is compact in $B(Y, Z)$.

The precise statement of the question we study in this paper is then as follows: is it true that for every $\varepsilon>0$ there exists a positive integer $k$ and points $\lambda_{i}$ of $\Delta$ such that $\left\|\sum_{i=1}^{k} c_{f, i} F_{\lambda_{i}}-P(\Delta) f\right\|_{Z} \leq \varepsilon\|f\|_{Y}$ for all $f \in Y$, where $c_{f, i}=\int_{0}^{\infty} f(x) F_{\lambda_{i}}(x) d x$ and where $F_{\lambda_{i}}$ is an eigenfunction in some sense of a given second-order ordinary differential operator $H$ on a half-line? The mapping taking $f$ to $c_{f, i} F_{\lambda_{i}}$ is the eigenprojection mentioned above.

Since it is known from [7] that the result is true if and only if $P(\Delta)$ is compact in $B(Y, Z)$, we ask the question for the optimal spaces $Y$ and $Z$ and expect the answer to depend on $\Delta$, since if the injection from $Y$ into $Z$ is compact it is known to be true for all $\Delta$. What should the spaces $Y$ and $Z$ be?

$Z$ should be the smallest space we can find which contains all the eigenfunctions; $Y$ should be contained in the dual space of $Z$ so that the integrals for the Fourier coefficients exist. What does it mean for $F_{\lambda}$ to be an eigenfunction?

If $\tau$ is a second-order ordinary differential expression on $(0, \infty)$, we will study the Dirichlet problem for $\tau$. This means at least that $\tau F_{\lambda}=\lambda F_{\lambda}$ and that $F_{\lambda}(0)=0$ and $F_{\lambda} \in Z$. We must define $Z$ to be the smallest space we can where there are enough $F_{\lambda}$ to at least perform the approximation one $f$ at a time. Fortunately, the general theory of continuous spectrum eigenfunctions gives us a good candidate for $Z$. Let $\tau$ be defined by $\tau \phi=-\phi^{\prime \prime}+q \phi$, and let $q$ be $C^{\infty}$, bounded below (this condition is relaxed in the body of the paper), and real valued. Define $Z$ as follows: let $\omega$ be an arbitrary bounded positive $C^{\infty}$ element of $L_{2}[0, \infty)$, and let $Z$ be $\left\{F: \omega F \in L_{2}[0, \infty)\right\}$; let the norm of $Z$ be given by $\|F\|_{Z}=\|\omega F\|_{2}$. It is shown in [7] that if $f / \omega \in L_{2}[0, \infty)$, then, for any Borel set $\Delta$, for every $\varepsilon>0$ there exists a positive integer $k$ and points $\lambda_{i}$ of $\Delta$ such that

$$
\left\|\sum_{i=1}^{k} c_{i, f} F_{\lambda_{i}}-P(\Delta) f\right\|_{Z}<\varepsilon\|f / \omega\|_{2},
$$

where $F_{\lambda_{i}} \in Z, \tau F_{\lambda_{i}}=\lambda_{i} F_{\lambda_{i}}, F_{\lambda_{i}}(0)=0$. This is our candidate for $Z$. Intuitively, $Z$ is a Hilbert space which is close to being $L_{\infty}[0, \infty)$, since $\omega$ can be an arbitrarily large fixed element of $L_{2}$. (Note, however, that the points $\lambda_{i}$ may depend upon $f$ in this statement, so the problem is not solved.) What is $Y$ ?

Optimally, we would like to take $Y$ to be $\left\{f: f / \omega \in L_{2}[0, \infty)\right\}$. However, if we do so, the injection from $Y$ into $Z$ is not compact. This indicates that the approximation property for this $Y$ depends on $\Delta$. For what sort of $\Delta$ would we expect success? Physical grounds lead to the suggestion that it should work if (and possibly only if) $\Delta$ is bounded. It is shown in [7] that the property holds if $\Delta$ is bounded, and also for some unbounded $\Delta$, at least when the spectrum is not wholly discrete. In this paper, our main result is Theorem 3.5 , which states that the approximation property holds if $(1+x) q \in L_{1}(0, x)$ and $\int_{\Delta}(1 / \sqrt{\lambda}) d \lambda<\infty$. 


\section{BACKGROUND}

Definition 1.1. Let $\tau$ be given by $\tau \phi=-\phi^{\prime \prime}+q \phi$ for all sufficiently differentiable $\phi$ on $(0, \infty)$, where $q$ is a $C^{\infty}$ function from $(0, \infty)$ into $\mathbb{R}$ and where $q=q_{+}+q_{-}$, with $q_{+}$and $q_{-}$being the positive and negative parts of $q$; assume that $q_{-}$is the sum of an $L_{\infty}$ function and an $L_{1}$ function.

Lemma 1.2. Under the above hypotheses, the restriction of $\tau$ to $C_{0}^{\infty}(0, \infty)$ is essentially selfadjoint. Let $H$ be the closure of this restriction. Then, for any $f$ in the domain of $H, f^{\prime} \in L_{2}[0, \infty)$.

Proof. This is a very special case of the results of Everitt, Hinton, and Wong [4].

Definition 1.3. Throughout this paper let $\omega$ be a fixed bounded positive $C^{\infty}(0, \infty)$ element of $L_{2}[0, \infty)$; let $Z_{\omega}$ denote $\left\{F: \omega F \in L_{2}\right\}$ and $Y_{\omega}$ denote $\left\{\phi: \phi / \omega \in L_{2}\right\}$; and let $\chi(\Delta)$ denote the characteristic function of the Borel set $\Delta$. Let $[$,$] denote the inner product in L_{2}[0, \infty)$.

Definition 1.4. Let $\Delta \rightarrow P(\Delta)$ be the spectral measure associated with $H ; \Delta$ ranges over the Borel subsets of $\mathbb{R}$. If $e \in L_{2}[0, \infty)$, let $S_{e}$ denote the closed linear span of $\{P(\Delta) e\}$, where $\Delta$ ranges over the Borel subsets of $\mathbb{R}$; let $\sigma_{e}$ be the positive measure on the Borel sets defined by $\sigma_{e}(\Delta)=[P(\Delta) e, e]$. Let $\Phi_{e}$ be the isometry given by the spectral theorem from $S_{e}$ onto $L_{2}\left(\sigma_{e}\right)$, which has the properties that $\Phi_{e}(P(\Delta) \phi)=\chi(\Delta) \Phi_{e}(\phi)$, for $\phi$ in $S_{e}$, and that $\Phi_{e}(H \phi)(\lambda)=$ $\lambda \Phi_{e}(\phi)(\lambda)$ for all $\phi$ in $D(H) \cap S_{e}$. Let $U_{e}=\Phi_{e} \circ P_{e}$, where $P_{e}$ is the orthogonal projection from $L_{2}[0, \infty)$ onto $S_{e}$.

Remark. Theorems 1.5 and 1.6 are proved in [7]; to apply the formalism of [7] let $W$ in [7] be $C_{0}^{\infty}(0, \infty)$ and let $B$ be multiplication by $\omega$. The results are stated in [7] only for $q$ bounded below, but the same proofs work for the $q$ of this paper.

Theorem 1.5. Suppose that $e$ is in the domain of $H$ and that $\|e\|_{h}^{2}=\|e\|^{2}+$ $\|H e\|^{2}$. Then there exists a subset $\zeta$ of $\mathbb{R}$ such that $\sigma_{e}(\mathbb{R} \backslash \zeta)=0$ and such that, for every $\lambda \in \zeta$, there exists a unique element $F_{\lambda, e}$ of $C^{\infty}[0, \infty)$ with the following properties:

(i) $\tau F_{\lambda, e}=\lambda F_{\lambda, e}$, and $F_{\lambda, e}(0)=0$; furthermore, for any $\theta \in C_{0}^{\infty}(0, \infty)$, $\int \theta(x) F_{\beta, e}(x) d x=U_{e}(\theta)(\beta)$ for almost every $\beta$ with respect to $\sigma_{e}$ (note that if $e \in W$, this implies that $\int e(x) F_{\lambda, e}(x) d x=1$, which may be regarded as a normalization of $\left.F_{\lambda, e}\right)$.

(ii) $F_{\lambda, e} \in Z_{\omega}$.

(iii) If $\alpha(\lambda)=F_{\lambda, e}$, then $\alpha$ is a measurable function with respect to $\sigma_{e}$ from $\mathbb{R}$ into $Z_{\omega}$, in the sense that $\forall \varepsilon>0$ there exists a compact set $\Gamma$ such that $\sigma_{e}(\mathbb{R} \backslash \Gamma)<\varepsilon$ and such that the restriction of $\alpha$ to $\Gamma$ is a continuous function from $\Gamma$ into $Z_{\omega}$.

(iv) If $\beta$ is a Borel subset of $[0, \infty)$ and $\Delta$ is a Borel subset of $\mathbb{R}$, then there is a positive constant $K$ such that $\int_{\Delta}\|\chi(\beta) \alpha(\lambda)\|_{Z_{\omega}} d \sigma_{e} \leq \sqrt{2} K\|P(\Delta) e\|_{h}\|\chi(\beta) \omega\|_{2}$.

Theorem 1.6. There exists an $e$ in the domain of $H$ such that $S_{e}=L_{2}$.

Remark. We now give the definition of diagonalization and introduce a spectral measure which we denote by $\mu_{e}$. Since the properties of $\mu_{e}$ are very important for our theory, it is useful to note that by an elementary calculation given below 
it follows that the definition of $\mu_{e}$ does not depend upon $e$ but only upon $S_{e}$. In other words, if $S_{e}=S_{f}$, then $\mu_{e}=\mu_{f}$.

Definition 1.7. Suppose that $e \in \operatorname{domain}(H)$ is a cyclic vector for $H$. Let $\mu_{e}$ be the positive measure on $\mathbb{R}$ defined by the relation $d \mu_{e}=\left\|F_{\lambda, e}\right\|_{Z_{\omega}}^{2} d \sigma_{e}$. Let $Q=r(H)$, where $r$ is a bounded continuous function from the spectrum of the restriction of $H$ to $S_{e}$ into $\mathbb{C}$. Let $\Delta$ be a Borel subset of $\mathbb{R}$. We say that $P(\Delta) P_{e} Q$ is diagonalizable in $B\left(Y_{\omega}, Z_{\omega}\right)$ with respect to $H$ if

(a) for every $\varepsilon>0$ there exists a positive integer $k$ and a finite disjoint family $\left\{\Delta_{i}\right\}_{i=1}^{k}$ of subsets of $\mathbb{R}$ such that $\mu_{e}\left(\Delta_{i}\right)$ is finite for every $i$ and such that there exists a set of real numbers $\left\{\lambda_{i}\right\}_{i=1}^{k}$ with $\lambda_{i} \in \Delta_{i} \cap \Delta$ and with the property that, injecting $P(\Delta) Q \theta$ canonically into $Z_{\omega}$,

$$
\left\|\left\{P(\Delta) P_{e} Q-\sum_{i=1}^{k} \mu_{e}\left(\Delta_{i} \cap \Delta\right) r\left(\lambda_{i}\right) R_{\lambda_{i}, e}\right\}(\theta)\right\|_{Z_{\omega}} \leq \varepsilon\|\theta\|_{Y_{\omega}}
$$

for all $\theta \in Y_{\omega}$, where

(b) $F_{\lambda_{i}, e} \in Z_{\omega}$ with $\left\|F_{\lambda, e}\right\|_{Z_{\omega}} \neq 0$, and where

(c) $R_{\lambda_{i}, e}(\phi)=G_{\lambda_{i}, e}(\phi) G_{\lambda_{i}, e}$ for any $\phi \in Y_{\omega}$, where $G_{\lambda_{i}, e}=F_{\lambda_{i}, e} /\left\|F_{\lambda_{i}, e}\right\|_{Z_{\omega}}$, and where $G_{\lambda_{i}}(\phi)$ is $\int_{0}^{\infty} \phi(x) G_{\lambda_{i}}(x) d x$. Note that while the points $\lambda_{i}$ depend on $\Delta$, the number $k$ and the sets $\Delta_{i}$ do not; these depend only upon $\varepsilon$.

Remark. The following is immediate from the fact that the norm limit of a sequence of operators with finite-dimensional range is compact.

Theorem 1.8. Suppose that $P(\Delta)$ is diagonalizable in $B\left(Y_{\omega}, Z_{\omega}\right)$ with respect to $H$. Then $P(\Delta)$ is a compact operator from $Y_{\omega}$ into $Z_{\omega}$. In particular, the identity operator $P(\mathbb{R})$ is not diagonalizable in $B\left(Y_{\omega}, Z_{\omega}\right)$ with respect to $H$.

Remark. Since the embedding of $Y_{\omega}$ into $L_{2}$ is continuous, as is the embedding of $L_{2}$ into $Z_{\omega}$, it follows that $P(\Delta)$ is in $B\left(Y_{\omega}, Z_{\omega}\right)$. Since the previous theorem showed that there exists an element $e$ of the domain of $H$ such that $S_{e}=L_{2}$, we do not need to consider $P_{e}$.

Remark. The following is part of Theorem 3.3 of [7], specialized to our situation.

Theorem 1.9. Suppose that $\mu_{e}$ is as in Definition 1.7 and that $\Delta$ is a Borel set such that $\mu_{e}(\Delta)$ is finite. Then $P(\Delta)$ is diagonalizable in $B\left(Y_{\omega}, Z_{\omega}\right)$.

Theorem 1.10. Let $\xi$ be a Borel subset of $\mathbb{R}$. Let $\phi(x, \lambda)$ be defined by $\tau \phi(x, \lambda)=\lambda \phi(x, \lambda), \phi(0, \lambda)=0$, and $\phi^{\prime}(0, \lambda)=1$. Let $\lambda \rightarrow J_{\lambda}$ be $a$ function with domain $\xi$ and range contained in the real-valued elements of $C^{\infty}([0, \infty))$, such that $J_{\lambda}(x)=a(\lambda) \phi(x, \lambda)$, with a measurable. Then the function $\lambda \rightarrow c_{\theta}(\lambda)=\int_{0}^{\infty} J_{\lambda}(x) \theta(x) d x$ is Borel measurable for any $\theta \in C_{0}^{\infty}(0, x)$. Let $\alpha$ be a positive Borel measure on $\xi$, such that for any subset $\Delta$ of $\xi$ and any $\theta \in C_{0}^{\infty}(0, \infty), c_{\theta}(\lambda) \in L_{2}(\alpha \mid \Delta)$ and $\|P(\Delta) \theta\|^{2}=\int_{\Delta}\left|c_{\theta}(\lambda)\right|^{2} d \alpha(\lambda)$. Then $\alpha$ and $\sigma_{e}$ are absolutely continuous with respect to each other, and hence $J_{\lambda} \in Z_{\omega}$ for almost every $\lambda$ with respect to $\alpha$. Furthermore, for any Borel subset $\Delta$ of $\xi$.

$$
\int_{\Delta}\left\|J_{\lambda}\right\|_{Z_{\omega}}^{2} d \alpha=\int_{\Delta}\left\|F_{\lambda, e}\right\|_{Z_{(j)}}^{2} d \sigma_{\rho}=\mu_{\rho}(\Delta) .
$$


Proof. First, note that by Theorem 1.5, $\int_{0}^{\infty} F_{\lambda, e}(x) \phi(x) d x=U_{e}(\phi)(\lambda)$ for almost every $\lambda$ with respect to $\sigma_{e}$. Hence, by the spectral theorem, $\|P(\Delta) \phi\|^{2}=$ $\int_{\Delta}\left|F_{\lambda, e}(\phi)\right|^{2} d \sigma_{e}(\lambda)$. By the spectral theorem, $\sigma_{e}$ is the unique such measure.

Now suppose that $J_{\lambda}$ and $\alpha$ are as above. Then $J_{\lambda}=b(\lambda) F_{\lambda, e}$, where $b$ is a measurable complex-valued function. Hence

$$
\|P(\Delta) \phi\|^{2}=\int_{\Delta}|b(\lambda)|^{2}\left|F_{\lambda, e}(\phi)\right|^{2} d \alpha(\lambda)
$$

for any $\Delta$. Hence $d \sigma_{e}=|b(\lambda)|^{2} d \alpha$. Now

$$
\int_{\Delta}\|J(\lambda)\|_{Z_{\omega}}^{2} d \alpha=\int_{\Delta}|b(\lambda)|^{2}\left\|F_{\lambda, e}\right\|_{Z_{\omega}}^{2} d \alpha=\int_{\Delta}\left\|F_{\lambda, e}\right\|_{Z_{\omega}}^{2} d \sigma_{e},
$$

as we desired to show.

Theorem 1.11. Suppose that $H$ has a purely discrete spectrum. Let $\left\{\psi_{i}\right\}$ be a complete orthonormal set of eigenfunctions of $H$, listed in increasing order of the eigenvalues $\lambda_{i}$. Let $\mu_{e}$ be as in Definition 1.7, with e a cyclic vector guaranteed by Theorem 1.6. Then $\mu_{e}(\Delta)=\sum_{\lambda_{i} \in \Delta}\left\|\psi_{i}\right\|_{Z_{\omega}}^{2}$.

Proof. Let $e$ be a cyclic vector as in Definition 1.7. $\sigma_{e}(\Delta)=\sum_{\lambda_{i} \in \Delta}\left|\left[\psi_{i}, e\right]\right|^{2}$. But $\left[e, F_{\lambda_{i}, e}\right]=1$. Thus $F_{\lambda_{i}, e}=\psi_{i} /\left[e, \psi_{i}\right]$. Hence $\mu_{e}(\Delta)=\int_{\Delta}\left\|F_{\lambda, e}\right\|_{Z_{i v}}^{2} d \sigma_{e}=$ $\sum_{\lambda_{i} \in \Delta}\left\|\psi_{i}\right\|_{Z_{\omega}}^{2}$, as we desired to show.

Remark. A simple direct argument using the above result shows that in the discrete spectrum case, $P(\Delta)$ is diagonalizable in $B\left(Y_{\omega}, Z_{\omega}\right)$ whenever $\mu_{e}(\Delta)<$ $\infty$. Hence Theorem 1.9 is only needed in the continuous spectrum case, which is the main case that we are interested in in this paper. In the discrete spectrum case, however, the question of whether unbounded sets $\Delta$ exist with $\mu_{e}(\Delta)<\infty$ is less easy. The fact that such sets do exist is a consequence of Theorem 2.2.

\section{THE DISCRETE SPECTRUM CASE}

The following estimates are fairly standard; see pp. 7-8 of [2], for example.

Lemma 2.1. There is a number $C$, independent of $x$ and $\lambda$, such that for $\lambda>0$ and $0 \leq x<\infty$, if $\phi(x, \lambda)$ is the solution to $\tau y=\lambda y, y(0)=0, y^{\prime}(0)=1$, and $\psi(x, \lambda)=x /(1+\sqrt{\lambda} x)$, then

(i) $|\phi(x, \lambda)| \leq C \psi(x, \lambda) \exp \left\{C \int_{0}^{x} \psi(s, \lambda)|q(s)| d s\right\}$;

(ii) $|\phi(x, \lambda)-\sin (\sqrt{\lambda} x) / \sqrt{\lambda}| \leq C \psi(x, \lambda)\left[\exp \left\{C \int_{0}^{x} \psi(s, \lambda)|q(s)| d s\right\}-1\right]$.

Theorem 2.2. Suppose the spectrum of $H$ is purely discrete. Suppose that $\omega$ is as in Definition 1.1 and that, in addition, $\lim _{x \rightarrow \infty} \omega(x)=0$. Let $\left\{\lambda_{i}\right\}$ be the sequence of eigenvalues, arranged in increasing order; and let $\left\{\psi_{i}\right\}$ be the sequence of associated eigenfunctions, normalized in $L_{2}$. Then $\lim _{n \rightarrow \infty}\left\|\psi_{n}\right\|_{Z_{w}}^{2}=$ $\lim _{n \rightarrow \infty}\left\{\int_{0}^{\infty} \omega^{2}(x) \psi_{n}^{2}(x) d x\right\}=0$.

Proof. With $\phi$ as above, $\psi_{n}(x)=d_{n} \phi\left(x, \lambda_{n}\right)$ for some sequence $d_{n}$ of constants. Part (ii) of the preceding lemma implies that if $\lambda_{n}>0$ and $x \in[0, b]$, then

$$
\begin{aligned}
& \left|\sqrt{\lambda_{n}} \phi\left(x, \lambda_{n}\right)-\sin \left(\sqrt{\lambda_{n}} x\right)\right| \\
& \quad \leq C\left(b \sqrt{\lambda_{n}} /\left(1+b \sqrt{\lambda_{n}}\right)\left[\exp \left\{C \int_{0}^{b}\left(s|q(s)| /\left(1+\sqrt{\lambda_{n}} s\right)\right) d s\right\}-1\right] .\right.
\end{aligned}
$$


It follows that $\left|\sqrt{\lambda_{n}} \phi\left(x, \lambda_{n}\right)-\sin \left(\sqrt{\lambda_{n}} x\right)\right|$ converges uniformly to 0 on $[0, b]$ as $n \rightarrow \infty$. Therefore,

$$
1=\int_{0}^{\infty} \psi_{n}(x)^{2} d x \geq \int_{0}^{b} \psi_{n}(x)^{2} d x=\int_{0}^{b} d_{n}^{2} \phi\left(x, \lambda_{n}\right)^{2} d x
$$

and

$$
\lim _{n \rightarrow \infty} \int_{0}^{b} \lambda_{n} \phi\left(x, \lambda_{n}\right)^{2} d x=\lim _{n \rightarrow \infty} \int_{0}^{b} \sin ^{2}\left(\sqrt{\lambda_{n}} x\right) d x=b / 2 .
$$

It follows from the preceding two inequalities that $\limsup _{n \rightarrow \infty} b d_{n}^{2} /\left(2 \lambda_{n}\right) \leq$ 1 . Since $b$ is arbitrary, it follows that $\lim _{n \rightarrow \infty}\left(d_{n}^{2} / \lambda_{n}\right)=0$. Therefore,

$$
\lim _{n \rightarrow \infty} \int_{0}^{b} \psi_{n}(x)^{2} d x=\lim _{n \rightarrow \infty}\left(d_{n}^{2} / \lambda_{n}\right) \int_{0}^{b} \lambda_{n} \phi\left(x, \lambda_{n}\right)^{2} d x=0 .
$$

But $\int_{b}^{\infty}\left|\omega \psi_{n}\right|^{2} \leq \sup _{x \geq b}|\omega(x)|^{2}\left\|\psi_{n}\right\|_{2}^{2}$. Using (2), we easily complete the proof.

Corollary 2.3. Under the hypotheses of Theorem 2.2, there exist infinite subsets $\Delta$ of the spectrum of $H$ on which $P(\Delta)$ is diagonalizable in $B\left(Y_{\omega}, Z_{\omega}\right)$.

Proof. This follows immediately from Theorems 2.2, 1.8, and 1.11.

\section{Decaying POtentials}

In this section, we assume, in addition, that $\int_{0}^{\infty}(1+x)|q(x)| d x<\infty$. Under this hypothesis, Theorems 3.1 and 3.2 are well known. Theorem 3.1 is well known in scattering theory. A proof can be found in [9, p. 185]. It also follows from the asymptotic behavior of the $m(\lambda)$ function at $\lambda=0$ and can be deduced from the results of Klaus [8]. Theorem $3: 2$ is a summary of results which appear in a variety of places. That (i) of Theorem $3: 2$ holds under the assumption that $q \in L_{1}$ appears in Titchmarsh [10] and in much more general circumstances in [5]. These references use the Titchmarsh-Kodaira formula to show that $\rho$ is continuously differentiable in $(0, \infty)$. Assertion (ii) follows from the asymptotics of the $m(\lambda)$ function given in [6] and the TitchmarshKodaira formula. Properties (iii) and (iv) are proved by Klaus. [8]. A standard reference for property (v) is Chapter 9 of [3].

Theorem 3.1. The restriction of the spectrum to $(-\infty, 0)$ consists of eigenvalues, which are finite in number (and which may be absent).

Theorem 3.2. There exists a continuously differentiable function $\rho(\lambda)$ with domain $(0, \infty)$, which has the following properties:

(i) $\rho$ is extendable to a continuous function on $[0, \infty)$;

(ii) $\lim _{\lambda \rightarrow \infty} \rho^{\prime}(\lambda) / \sqrt{\lambda}=c_{0}>0$;

(iii) if $\phi(x, 0) \notin L_{\infty}[0, \infty)$, then $\lim _{\lambda \rightarrow 0^{+}} \rho^{\prime}(\lambda) / \sqrt{\lambda}=a_{0}>0$;

(iv) if $\phi(x, 0) \in L_{\infty}[0, \infty)$, then $\lim _{\lambda \rightarrow 0^{+}} \sqrt{\lambda} \rho^{\prime}(\lambda)=b_{0} \neq 0$;

(v) for any $\theta \in C_{0}^{\infty}(0, \infty)$, defining $F(\lambda)$ to be $\int_{0}^{\infty} \theta(x) \phi(x, \lambda) d x$, then for each positive integer $N, P(0, N] \theta(x)=\int_{(0, N]} F(\lambda) \phi(x, \lambda) \rho^{\prime}(\lambda) d \lambda$. (This property uniquely specifies $\rho^{\prime}$.) 
Theorem 3.3. Suppose that $\Delta \subset \mathbb{R}$ and that either 0 is not a limit point of $\Delta$ or $\phi(x, 0) \notin L_{\infty}[0, \infty)$. Suppose also that $\int_{\Delta}(1 / \sqrt{\lambda}) d \lambda<\infty$. Then $\mu_{e}(\Delta)$ is finite.

Proof. Since spectrum $(H) \cap(-\infty, 0]$ is a finite set, it follows from Theorems 1.3 and 1.9 that this set has finite measure with respect to $\mu_{e}$. Hence we need only consider the case where $\Delta$ is a subset of $(0, \infty)$. Define $J_{\lambda}(x)$ to be $\phi(x, \lambda)$. Then $J_{\lambda}$ satisfies the hypotheses of Theorem 1.10. Hence

$$
\mu_{e}(\Delta)=\int_{\Delta}\|\phi(x, \lambda)\|_{Z_{\omega}}^{2} \rho^{\prime}(\lambda) d \lambda=\int_{\Delta}\left\{\int_{0}^{\infty} \omega^{2} \phi(x, \lambda)^{2} d x\right\} \rho^{\prime}(\lambda) d \lambda .
$$

Since $\int_{0}^{\infty}(1+x)|q(x)| d x<\infty$, it follows from part (i) of Lemma 2.1 that $|\phi(x, \lambda)| \leq K x /(1+\sqrt{\lambda} x)$, where $K$ is independent of $x$ and $\lambda$. Hence, for $\Delta \subset[0, \infty)$,

$$
\begin{aligned}
\mu_{e}(\Delta) & \leq \int_{\Delta}\left\{\int_{0}^{\infty}\left\{\omega^{2}(x) K^{2} x^{2} /(1+\sqrt{\lambda} x)^{2}\right\} d x\right\} \rho^{\prime}(\lambda) d \lambda \\
& \leq \int_{\Delta}\left\{\int_{0}^{\infty} \omega^{2}(x)\left(K^{2} / \lambda\right) d x\right\} M \sqrt{\lambda} d \lambda .
\end{aligned}
$$

The last inequality follows from the fact that $\rho^{\prime}(\lambda) \leq M \sqrt{\lambda}$, which follows from Theorem 3.2. From (3), the theorem is obvious.

Theoxem 3.4. Suppose that $\phi(x, 0) \in L_{\infty}[0, \infty)$ and that 0 is a limit point of $\Delta$. Then $\mu_{e}(\Delta)<\infty$ if $\int_{\Delta}(1 / \sqrt{\lambda}) d \lambda<\infty$, provided the additional hypothesis $\int_{0}^{\infty} x^{2} \omega^{2}(x) d x<\infty$ holds.

Proof. Let $\Delta=\Delta_{1} \cup \Delta_{2}$, where $\Delta_{1}=\Delta \cap(0,1)$. By the preceding theorem, $\mu_{e}\left(\Delta_{2}\right)<\infty$. Using the first inequality of (3), we see that

$$
\begin{aligned}
\mu_{e}\left(\Delta_{1}\right) & \leq \int_{\Delta_{1}}\left\{\int_{0}^{\infty} \omega^{2}(x) K^{2} x^{2} /(1+\sqrt{\lambda} x)^{2} d x\right\} \rho^{\prime}(\lambda) d \lambda \\
& \leq\left(\int_{0}^{\infty} K^{2} x^{2} \omega^{2}(x) d x\right) \rho\left(\Delta_{1}\right) .
\end{aligned}
$$

The result follows.

Theorem 3.5. Suppose that $\Delta$ is a subset of $\mathbb{R}$ such that $\int_{\Delta \cap[1, \infty)}(1 / \sqrt{\lambda}) d \lambda<\infty$. Then $P(\Delta)$ is diagonalizable in $B\left(Y_{\omega}, Z_{\omega}\right)$, and therefore $P(\Delta)$ is compact in $B\left(Y_{\omega}, L_{2}\right)$.

Remark. Compactness in $B\left(Y_{\omega}, L_{2}\right)$ is seemingly stronger than compactness in $B\left(Y_{\omega}, Z_{\omega}\right)$, since $L_{2}$ is topologically embedded in $Z_{\omega}$. For $P(\Delta)$, however, the two are equivalent by Theorem 5.2 of [7].

Proof. The result follows from Theorem 3.3, combined with the observation that if $P(\Delta)$ is diagonalizable on each of two sets it is diagonalizable on their union. It should be noted that $P(\Delta)$ is diagonalizable on any bounded set by Theorem 5.5 of [7].

Example. To get some perspective on the results of this section, let us consider the special case where $q(x)=0$. In this case the usual Fourier sine transform 
may be written for $f \in L_{2}[0, \infty)$ and any Borel set $\Delta$ :

$$
\begin{aligned}
& \text { (a) } \mathscr{F}(\lambda)=\int_{0}^{\infty}(\sin (\sqrt{\lambda} x) / \sqrt{\lambda}) f(x) d x, \\
& \text { (b) } P(\Delta) f(x)=\int_{\Delta} \mathscr{F}(\lambda)(\sin (\sqrt{\lambda} x) / \sqrt{\lambda}) d \rho(\lambda),
\end{aligned}
$$

where $\rho(\lambda)=2 \lambda^{3 / 2} / 3 \pi$. The convergence of (a) is in the norm of $L_{2}(\rho)$, and that of $(b)$ is in the norm of $L_{2}[0, \infty)$; what this means is that the restriction of $\mathscr{F}$ to $C_{0}^{\infty}(0, \infty)$ extends by continuity to a unitary operator from $L_{2}[0, \infty)$ onto $L_{2}(\rho)$, although for arbitrary $f$ neither integral exists in the usual sense. Choose $e$ so that $S_{e}=L_{2}[0, \infty)$. Since $q(x)=0, F_{\lambda, e}(x)=b(\lambda) \sin (\sqrt{\lambda} x)$ for $\lambda>0$, and by Theorem 1.10 , with $\alpha=\rho$,

$$
\text { (c) } \begin{aligned}
\mu_{e}(\Delta) & =\int_{\Delta}\|\sin (\sqrt{\lambda} x) / \sqrt{\lambda}\|_{Z_{\omega}}^{2} d \rho(\lambda) \\
& =\int_{\Delta}\left(\int_{0}^{\infty} \sin ^{2}(\sqrt{\lambda} x) \omega^{2}(x) d x\right)(1 / \pi \sqrt{\lambda}) d \lambda .
\end{aligned}
$$

Furthermore, if $c_{\theta}(\lambda)=\int_{0}^{\infty} F_{\lambda, e}(x) \theta(x) d x$,

(d) $c_{\theta}\left(\lambda_{i}\right) F_{\lambda_{i}, e}(s) /\left\|F_{\lambda_{i}, e}\right\|_{Z_{w}}^{2}$

$$
=\left[\int_{0}^{\infty} \sin \left(\sqrt{\lambda_{i}} x\right) \theta(x) d x\right] \sin \left(\sqrt{\lambda_{i}} s\right) / \int_{0}^{\infty} \omega(x)^{2} \sin ^{2}\left(\sqrt{\lambda_{i}} x\right) d x .
$$

By Theorem 1.9, if $\mu_{e}(\Delta)<\infty$ and $\varepsilon>0$, there are sets $\Delta_{1}, \ldots, \Delta_{k}$ and points $\lambda_{i} \in \Delta_{i} \cap \Delta$ such that for any $\theta \in Y_{\omega}$

$$
\left\|P(\Delta) \theta-\sum_{i=1}^{k} \mu_{e}\left(\Delta \cap \Delta_{i}\right) c_{\theta}\left(\lambda_{i}\right) F_{\lambda_{i}, e} /\right\| F_{\lambda_{i}, e}\left\|_{Z_{\omega}}^{2}\right\|_{Z_{\omega}} \leq \varepsilon\|\theta\|_{Y_{\omega}} .
$$

This fact also follows in this context directly from the continuity of the mapping taking $\lambda$ to $\sin (\sqrt{\lambda} x) / \sqrt{\lambda}$, considered as a function from $\mathbb{R}$ into $Z_{\omega}$. This continuity does not hold in general, though it does hold when $(1+x) q \in L_{1}$. A good deal of the work involved in proving Theorem 1.9 is to compensate for this lack of continuity. Note that $b(\lambda)$ plays no role in the calculations.

For example, if $\omega=1 /\left(1+x^{2}\right)^{1 / 2}$,

$$
\int_{0}^{\infty} \sin ^{2}(\sqrt{\lambda} x) \omega^{2}(x) d x=\pi\left(1-e^{-2 \sqrt{\lambda}}\right) / 4
$$

so that $\mu_{e}(\Delta)=\int_{\Delta} \frac{1}{4}\left(1-e^{-2 \sqrt{\lambda}}\right) d \lambda / \sqrt{\lambda}$.

If $\omega=e^{-(x / 2)}$, then $\int_{0}^{\infty} \sin ^{2}(\sqrt{\lambda} x) \omega^{2}(x) d x=2 \lambda /(1+4 \lambda)$. Hence in this case $\mu_{e}(\Delta)=\int_{\Delta}[2 \sqrt{\lambda} /(\pi+4 \pi \lambda)] d \lambda$.

Note that in each case for $\mu_{e}(\Delta)$ to be finite it must be true that $\int_{\Delta}(1 / \sqrt{\lambda}) d \lambda$ $<\infty$. Note also that the measure $\mu_{e}$ is quite different from the measure $\rho$.

\section{REFERENCES}

1. F. V. Atkinson, On the asymptotic behavior of the Titchmarsh-Weyl m-coefficient and the spectral function for scalar second-order differential expressions, Lecture Notes in Math., vol. 964, Springer-Verlag, Berlin, Heidelberg, and New York, 1982, pp. 1-27. 
2. K. Chadan and P. C. Sabatier, Inverse problems in quantum scattering theory, SpringerVerlag, Berlin, Heidelberg, and New York, 1989.

3. E. A. Coddington and N. Levinson, Theory of ordinary differential equations, McGraw-Hill, New York, 1965.

4. W. N. Everitt, D. B. Hinton, and J. S. W. Wong, On the strong limit-n classification of linear ordinary differential expressions of order 2n, Proc. London Math. Soc. (3) 29 (1974), 351357.

5. D. B. Hinton and J. K. Shaw, Absolutely continuous spectra of second order differential operators with short and long range potentials, SIAM J. Math. Anal. 17 (1986), 182-196.

6. D. B. Hinton, M. Klaus, and J. K. Shaw, Series representation and asymptotics for Titchmarsh-Weyl $m$ functions, Differential Integral Equations 2 (1989), 419-429.

7. R. M. Kauffman, Finite eigenfunction approximation for continuous spectrum operators, Internat. J. Math. Math. Sci. (to appear).

8. M. Klaus, On the variation-diminishing property of Schrödinger operators, Oscillation, Bifurcation, and Chaos, CMS Conference Proceedings, vol. 8, Amer. Math. Soc., Providence, RI, 1986, pp. 199-212.

9. V. A. Marchenko, Sturm-Liouville operators and applications, Birkhäuser Verlag, Boston, MA, 1986.

10. E. C. Titchmarsh, Eigenfunction expansions, Part 1, Oxford Univ. Press, Oxford, 1962.

Department of Mathematics, University of Tennessee, Knoxville, Tennessee 37996

E-mail address: IN\%Hinton@UTKVX.bitnet

Department of Mathematics, University of Alabama at Birmingham, Birmingham, AlABAMA 35294

E-mail address: KAUFFMAN@math.uab.edu 